\title{
Molecular method for the characterization of Coxiella burnetii from clinical and environmental samples: variability of genotypes in Spain
}

Isabel Jado ${ }^{*}$, Cristina Carranza-Rodríguez ${ }^{2}$, Jesús Félix Barandika ${ }^{3}$, Álvaro Toledo ${ }^{4,9}$, Cristina García-Amil ${ }^{1}$, Beatriz Serrano ${ }^{5}$, Margarita Bolaños ${ }^{2}$, Horacio Gil ${ }^{1}$, Raquel Escudero ${ }^{1}$, Ana L García-Pérez ${ }^{3}$, A Sonia Olmeda ${ }^{4}$, lanire Astobiza ${ }^{3}$, Bruno Lobo ${ }^{1}$, Manuela Rodríguez-Vargas ${ }^{1}$, José Luis Pérez-Arellano ${ }^{2}$, Fernando López-Gatius ${ }^{5}$, Francisco Pascual-Velasco ${ }^{6}$, Gustavo Cilla ${ }^{7}$, Noé F Rodríguez ${ }^{8}$ and Pedro Anda ${ }^{1}$

\begin{abstract}
Background: Coxiella burnetii is a highly clonal microorganism which is difficult to culture, requiring BSL3 conditions for its propagation. This leads to a scarce availability of isolates worldwide. On the other hand, published methods of characterization have delineated up to 8 different genomic groups and 36 genotypes. However, all these methodologies, with the exception of one that exhibited limited discriminatory power (3 genotypes), rely on performing between 10 and 20 PCR amplifications or sequencing long fragments of DNA, which make their direct application to clinical samples impracticable and leads to a scarce accessibility of data on the circulation of C. burnetii genotypes.

Results: To assess the variability of this organism in Spain, we have developed a novel method that consists of a multiplex (8 targets) PCR and hybridization with specific probes that reproduce the previous classification of this organism into 8 genomic groups, and up to 16 genotypes. It allows for a direct characterization from clinical and environmental samples in a single run, which will help in the study of the different genotypes circulating in wild and domestic cycles as well as from sporadic human cases and outbreaks. The method has been validated with reference isolates. A high variability of $C$. burnetii has been found in Spain among 90 samples tested, detecting 10 different genotypes, being those adaA negative associated with acute $\mathrm{Q}$ fever cases presenting as fever of intermediate duration with liver involvement and with chronic cases. Genotypes infecting humans are also found in sheep, goats, rats, wild boar and ticks, and the only genotype found in cattle has never been found among our clinical samples.

Conclusions: This newly developed methodology has permitted to demonstrate that $C$. burnetii is highly variable in Spain. With the data presented here, cattle seem not to participate in the transmission of $C$. burnetii to humans in the samples studied, while sheep, goats, wild boar, rats and ticks share genotypes with the human population.
\end{abstract}

\section{Background}

Coxiella burnetii is an obligate intracellular Gram negative bacterium which causes $\mathrm{Q}$ fever, an illness with multiple clinical manifestations in its acute presentation, including a flu-like respiratory process that could result in atypical pneumonia, or fever of intermediate duration (FID) with liver involvement. In a low percentage of cases a chronic form of the disease is diagnosed, characterized by

\footnotetext{
* Correspondence: ijado@isciii.es

'Laboratorio de Espiroquetas y Patógenos Especiales, Department of Bacteriology, Centro Nacional de Microbiología, Instituto de Salud Carlos III, Ctra. de Pozuelo km 2.6, Majadahonda, Madrid 28220, Spain

Full list of author information is available at the end of the article
}

an infection that persists for more than 6 months, more frequently endocarditis, which can be fatal without an appropriate treatment [1]. Its high infectivity, resistance in adverse environmental conditions and aerosol route of transmission make this agent a candidate for intentional release [2], being listed as a category B bioterrorism agent by the USA Centers for Disease Control and Prevention.

Initial studies tried to correlate specific genotypes (GT) with the chronic and acute forms of the disease. Thus, certain plasmid patterns were claimed to be associated with the disease outcome [3,4], which was controversial [5]; also, some isocitrate dehydrogenase types were associated with chronic disease and a role for this gene in the

\section{() Biomed Central}


adaptation of the organism to the intracellular environment was proposed [6], although this association was also challenged by other authors [7].

More recently, different attempts have been made to classify isolates of $C$. burnetii in different genomic groups (GG). Based on restriction fragment length polymorphism (RFLP) of the entire genome, Hendrix et al. [8] resolved 36 isolates of different origin in 6 GG; Jager et al. [9] performed pulsed field gel electrophoresis (PFGE) in 80 isolates that were classified into 4 GG; a Multispacer Sequence Typing method [10], based on the sequencing of 10 intergenic spacers classified 173 isolates, mainly from chronic disease, into 3 monophyletic groups and $30 \mathrm{GT}$; later, a reduced MST method was published by Mediannikov et al. [11], targeting 3 spacers in a single PCR, detecting 3 MST GTs; Svraka et al. [12] amplified different variable number tandem repeats (VNTR) in 7 PCR protocols, describing 5 clusters and validating previous GG ascriptions; Arricau-Bouvery [13] applied infrequent restriction site-PCR and multilocus variable number of tandem repeats analysis (MLVA) for the typing in a scheme of 17 PCR protocols leading to a classification into 6 clusters and 36 GT; later, Roest et al. [14], used the same method but reducing the 17 described targets to 10 , to study an outbreak in the Netherlands and describing 13 MLVA types; Beare et al. [15] added two more GG, totalling up to 8 , in a microarray-based whole genome comparison; Denison et al. [16] performed 20 PCRs for the characterization of the region within and near the transposase IS1111, describing 5 GG among 21 reference strains and 9 animal samples; Huijsmans et al. [17] developed a method for a singlenucleotide-polymorphisms (SNP)-based typing, applying 10 real time PCR protocols that resolved 28 reference strains and 40 samples from an outbreak into 9 SNP genotypes, while a previous study on the same 28 reference strains [13] had disclosed 14 MLVA types; finally, Hornstra et al. [18] performed 14 SNP-based real time PCR assays that classified 63 isolates into 6 GG and 35 MST genotypes.

Recently, an outer membrane protein-coding gene named acute disease antigen $A(\operatorname{ada} A)$ was described as associated with acute $\mathrm{Q}$ fever-causing strains, whereas adaA negative strains were linked to chronic cases [19]. Therefore, the hypothesis of its association with a specific clinical presentation of the disease together with its immunodominant nature lead the authors to suggest that $a d a A$ may be a virulence factor for the pathogenesis of $\mathrm{Q}$ fever. Consequently, adaA may be a relevant genetic marker for differentiation among isolates.

In general, there has been a good correlation between typing methods although with different discriminatory capabilities. However, although 2 previous descriptions have been applied directly to clinical samples $[16,17]$, both rely on the amplification of several targets performing between 10 and 20 PCR protocols, which make it not always feasible for their use in a clinical setting due to the frequent scarcity of testable sample-size, which hampers the acquisition of global data; the method of Mediannikov et al. [11], consisting of a multiplex PCR targeting 3 intergenic spacers, exhibited however a limited discriminatory power (3 MST types) in the samples studied. In this study, based on the previous descriptions of Beare et al. [15] and Zhang et al. [19], a fast, reproducible and sensitive multiplex PCR that amplifies 8 targets in the same run for a rapid GT determination, has been developed to be applied to both isolates and PCR-positive samples. With this method, C. burnetii could be classified into 8 GG and up to 16 GT. Based on this methodology, a comprehensive study on the variability of $C$. burnetii in Spain have been made with samples from patients with acute and chronic Q fever, domestic and wild mammals and ticks, demonstrating a high variability of this organism and an association between genotypes and human disease.

\section{Methods \\ Samples}

Fifteen $C$. burnetii reference isolates were used to validate the typing method (Additional file 1: Table S1). Also, human and animal samples (livestock, wild animals and ticks) sent to the National Reference Laboratory at the Instituto de Salud Carlos III and to the clinical and veterinarian collaborating laboratories for diagnosis of $\mathrm{Q}$ fever were included in the study, including defibrinated blood, plasma, biopsy material, ruminant placentas, mostly from abortions with the exception of 3 cattle placentas from normal parturitions (Additional file 1: Table S1), and other tissues from domestic and wild animals, and questing ticks, that were collected from different areas in Central Spain: 4 areas in Madrid (Cercedilla, Aranjuez, Perales and Valdeolmos) and 1 in Toledo (Oropesa). In all the areas the presence of livestock was documented (cattle in all areas and sheep and swine only in Oropesa). There were remarkable high densities of rabbits (Oryctolagus cuniculus) in all the areas except Cercedilla. The study protocol was approved by the Bioethics and Animal Welfare Committee of the Instituto de Salud Carlos III, Spain (ref. CBBA/4 2006), where the study was conducted, respecting individual privacy according to relevant data protection legislation and animal welfare. Also, human clinical samples used in the study were made available to us in an anonymized manner.

\section{Culture}

Standard shell-vial methodology was used as previously described [20] to grow C. burnetii in Vero E6 cells (European Collection of Cell Cultures; provided by Sigma-Aldrich Química S.A., Tres Cantos, Madrid, Spain). All the propagative methods and those related to 
the manipulation of domestic ruminant placentas were performed under Biosafety level 3 (BSL3) conditions.

\section{Molecular detection of C. burnetii}

DNA was extracted from samples and isolates with the Qiagen Tissue kit (IZASA S.A. Barcelona, Spain). For arthropods, specimens were first crushed in $1.5 \mathrm{ml}$ eppendorf tubes with the help of a pestle (Sigma-Aldrich Química S.A., Barcelona, Spain), as described [21], and extracted as before. DNA was quantified in a NanoDrop ND-1000 spectrophotometer (NanoDrop Technologies Inc. Wilmington, Delaware USA), and about 200 ng were used for each PCR. Previous to the genotyping, a screening assay (IS1111-based PCR coupled with hybridization with a specific probe by reverse line blotting -RLB) was used for the detection of C. burnetii [22-24].

\section{C. burnetii genotyping}

An analysis based on a previous report [15] was performed to identify which genes/ORFs defined the ascription of each isolate to a specific GG, and seven of them were selected (CBU0007, CBU0071, CBU0168, CBU0598, CBU0881, CBU1805 and CBU2026), whose combination of presence/absence seems to determine the GG (Table 1). Also, the detection of adaA (CBU0952) [19] was included in the method. Primers and probes were designed for their molecular detection by PCR and subsequent RLB hybridization (Additional file 2: Table S2) by using Oligo6 software (Molecular Biology Insights, Inc. Cascade, CO, USA). Incompatibility among primers was avoided by in silico analysis of the formation of secondary structures, and oligonucleotides forming dimers with energy values lower than $-6 \mathrm{kcal} / \mathrm{mol}$ and hairpins with Tm higher than $40 \mathrm{C}$ were discarded. The specificity of the oligonucleotides was first assessed by blastn (http://www.ncbi.nlm.nih.gov/ blast/Blast.cgi?PAGE=Nucleotides). The reaction mix included $80 \mu \mathrm{g} /$ tube of bovine serum albumin (Roche España, Madrid, Spain), $3.75 \mathrm{mM} \mathrm{MgCl}_{2}$ (Applied Biosystems), $200 \mu \mathrm{M}$ dNTPs (Applied Biosystems) and $4 \mathrm{U}$ of AmpliTaq Gold ${ }^{\circledR}$ DNA Polymerase (Amersham Pharmacia Biotech, Cerdanyola del Vallès, Barcelona, Spain). Primer

Table 1 Scheme of the presence/absence of the Coxiella burnetii ORFs selected for the determination of genomic groups

\begin{tabular}{lcccccccc}
\hline Target & GGI & GGII & GGIII & GGIV & GGV & GGVI & GGVII & GGVIII \\
\hline CBU0007 & + & + & + & - & + & + & + & + \\
\hline CBU 0071 & + & + & + & + & - & + & + & - \\
\hline CBU 0168 & + & + & + & - & + & + & - & + \\
\hline CBU 0598 & + & + & - & + & + & + & + & + \\
\hline CBU 0881 & + & + & + & + & + & - & - & - \\
\hline CBU 1805 & + & + & + & + & - & + & + & + \\
\hline CBU 2026 & + & - & + & + & + & + & + & + \\
\hline
\end{tabular}

concentrations ranged from 0.6 to $1 \mu \mathrm{M}$ (Additional file 2: Table S2). The amplification cycles included an initial cycle of $94 \mathrm{C}$ for $9 \mathrm{~min}$, followed by 40 cycles of $94 \mathrm{C} 30 \mathrm{~s}, 60 \mathrm{C}$ $1 \mathrm{~min}$, and $72 \mathrm{C} 1 \mathrm{~min}$, with a final extension at $72 \mathrm{C}$ for $10 \mathrm{~min}$. The amplifications were performed in an $\mathrm{MJ}$ Research PTC-200 (Bio-Rad Laboratories, S.A., Alcobendas, Madrid, Spain) in volumes of $50 \mu \mathrm{l}$. Hybridization by RLB was performed as described [25] using $48 \mathrm{C}$ for the hybridization and $40 \mathrm{C}$ for the conjugate and the washing steps. Concentration of probes ranged from 0.8 to 6.4 pmols/ $\mu$ l (Additional file 2: Table S2). Two overlapping films (SuperRX, Fujifilm España S.A., Barcelona, Spain), were used in each assay to obtain a less and more exposed image for each membrane.

The sensitivity of the technique was checked with serial 10 -fold dilutions of a purified DNA stock of the isolate Nine Mile phase II (NMII) and the specificity was studied by subjecting to the method $10^{4}$ genome equivalents of a selection of other bacterial species causing zoonoses or related illness (Orientia tsutsugamushi, Rickettsia conorii, $R$. typhi, Legionella pneumophila, Francisella tularensis subsp. holarctica, Bartonella henselae, Chlamydophila pneumoniae, and Mycoplasma pneumoniae).

To assess the reproducibility of the methodology, DNA extracted from 2 different passages ( $n$ and $n+10$ ) of 5 reference isolates (NMI, CS-27, Priscilla, SQ217, F2) and a local isolate from cattle (273) (Additional file 1: Table S1) were analyzed.

The results of the GT study were further analyzed by using InfoQuest FP 4.50 (BioRad, Hercules, CA, USA). Clustering analyses used the binary coefficient (Jaccard) and UPGMA (Unweigthed Pair Group Method Using Arithmetic Averages) to infer the phylogenetic relationships.

\section{Results}

\section{Samples included in the study}

A total of 90 autochthonous samples were included: 24 from human patients (11 from acute and 13 from chronic cases) and 66 from animals and ticks (15 from sheep, 7 from goats, 7 from cattle, 3 from rats, 1 from a wild boar and 33 from ticks); also, 15 reference strains were used to validate the method (Additional file 1: Table S1).

\section{Sensitivity, specificity and reproducibility of the method} The detection limit of the method using purified NMII DNA was 10 genome equivalents. No reactivity was detected when testing $10^{4}$ genome equivalents from 8 other bacterial species causing zoonoses or related illness (data not shown). To check for reproducibility, passages "n" (Figure 1, lanes 1-6) and "n+10" (Figure 1, lanes 7-12) from 5 reference isolates and a local isolate from cattle were checked without any loss of sensitivity during in vitro 


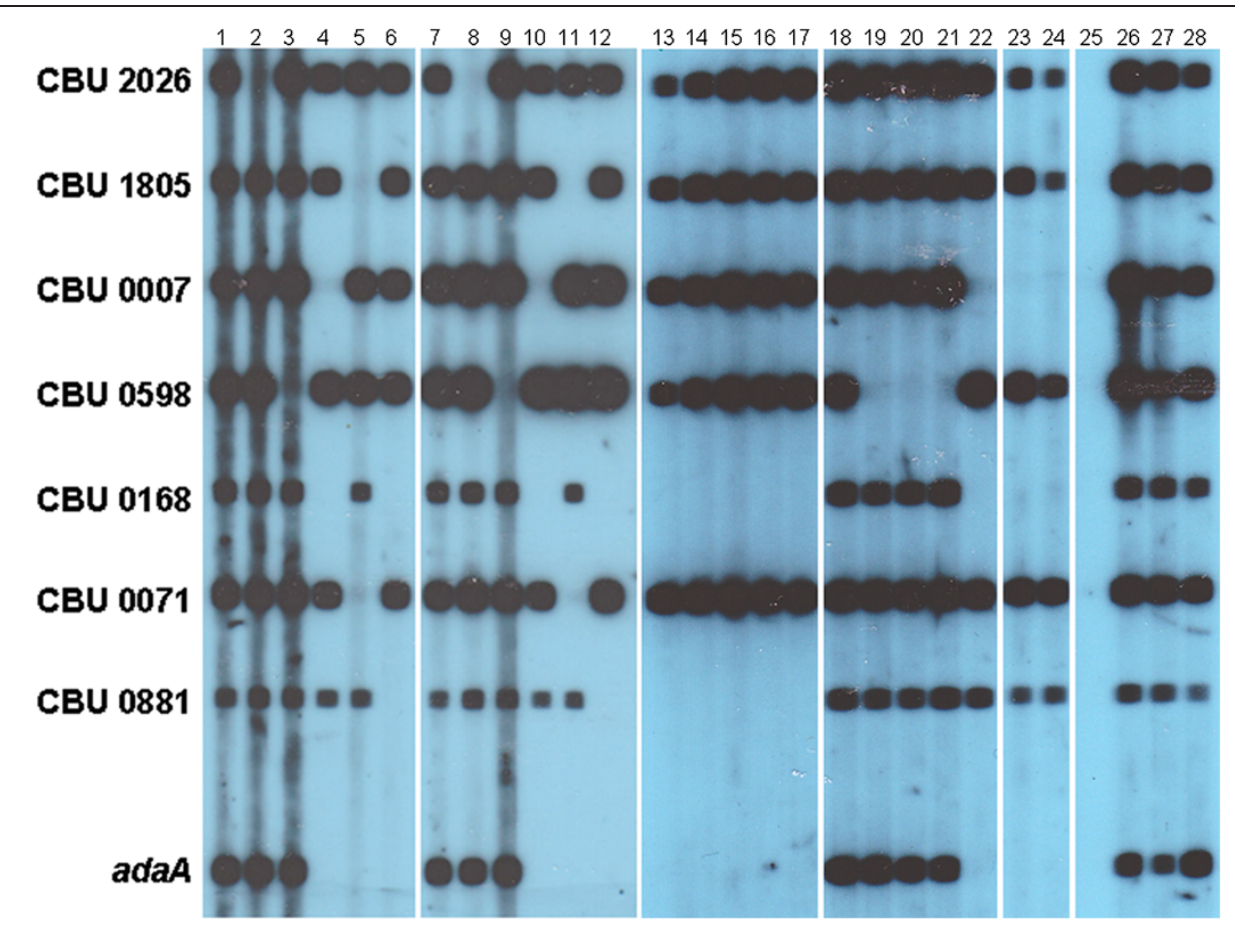

Figure 1 Sensitivity and reproducibility of the method of characterization of Coxiella burnetii. Reproducibility. Lanes 1-6: Isolates NMI, CS-27, local cattle isolate 273, Priscilla, SQ217 and F2, passage " $n$ "; lanes 7-12: same isolates, passage " $n+10$ ". Results using clinical, veterinary and arthropod samples. Lane 13: specimen of R. sanguineus (M28CE4GA7C); lanes 14-16: specimens of H. lusitanicum (M28P1GA8A, M28PE14GV5C and M28PE14GV5F); lane 17: specimen of D. marginatus (M28P2GA45C); lane 18: human serum (2172); lane 19: sheep placenta (70924); lane 20: goat lung (67025); lane 21: cattle endocervical exudate (70814); lane 22: human clot (BZO18); lane 23: human plasma (0904); lane 24: rat liver (78); lane 25: negative control. Sensitivity. Lanes 26-28: $10^{3}, 10^{2}$ and 10 genome equivalents of isolate NMII. Left panel: position of the probes for each ORF.

passages in any of the targets assayed. Also, it is to note that NMI (phase I) and NMII (phase II) isolates presented the same results in this characterization; consequently, only one of them (NMI) was used throughout the study.

\section{Genotyping of reference isolates and samples}

From the 15 reference isolates that were tested with the method described here for GG adscription (Table 1), and from which data from previous studies were available, all of them fell in the same GG as previously described, the topology of the tree being consistent with previous data. None of the reference isolates tested was found to belong to GG III, VI and VIII (Additional file 1: Table S1, Figure 2).

Local human samples were found to belong to GG I, IV, VII and VIII, as follows: 13 samples from chronic cases (7 endocarditis, 3 vascular infections, 1 infected aneurism, 1 osteomyelitis and 1 chronic hepatitis) from 8 different regions were all infected with GG IV, except for one vascular infection (GG VIII); acute cases (10 samples of FID with liver involvement and 1 sample of pneumonia; 4 regions) showed GG I, IV, VII and VIII (Additional file 1: Table S1, Table 2, Figures 2 and 3).
Among livestock samples, those from sheep (15 samples from 8 provinces) were found belonging to GG I, II, III, IV and VIII; goats (7 samples from 4 provinces) were infected with GG III, IV and VIII; cattle (7 samples from 4 provinces) were all infected by GG III; rats (3 samples from 1 province) and a wild boar showed GG IV; finally, 33 ticks of 3 species, from 4 areas of 2 adjacent regions, carried always GG VII, except for one that carried GG VI. In summary, samples from GG I, II, III, IV, VI, VII and VIII were identified (Additional file 1: Table S1; Table 2, Figures 2 and 3).

\section{adaA detection}

Samples from GG I, II and III were always $a d a A$ positive; all GG IV were adaA negative, except for a sheep placenta that was adaA positive; GG VII samples were adaA negative, except for a tick specimen; GG VIII samples were positive, except for a human sample of acute hepatitis; finally, the only sample available from GG VI (one H. lusitanicum tick) was adaA negative (Additional file 1: Table S1, Table 2, Figure 2). All the samples from cases of acute FID with liver involvement (10 samples from 3 distant regions; Figure 3) were $a d a A$ negative and the only sample available from a patient with pneumonia was $a d a A$ positive. 


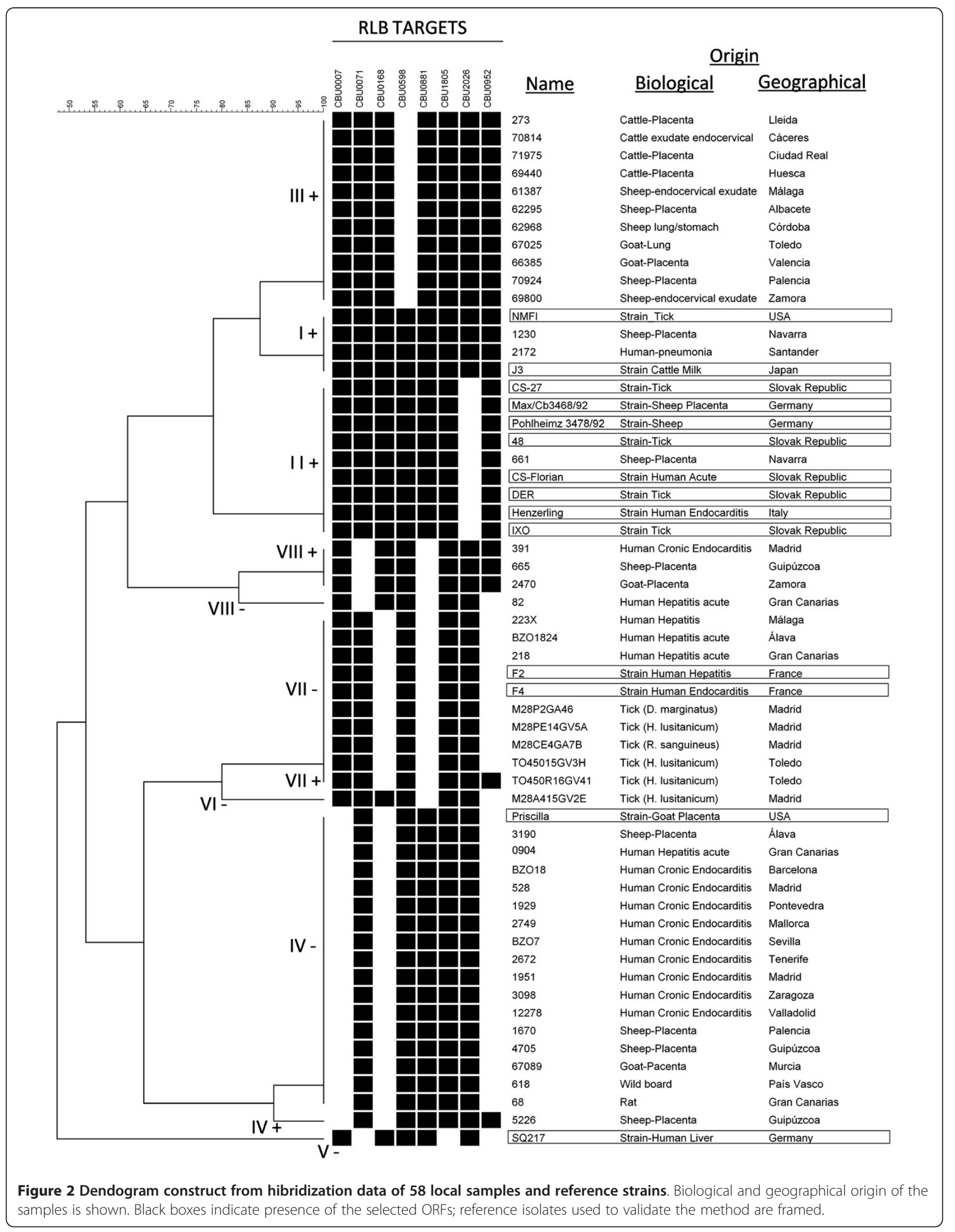


Table 2 Summary of the results of the characterization

\begin{tabular}{|c|c|c|c|c|c|c|c|c|c|}
\hline \multirow[t]{2}{*}{ GT } & \multicolumn{3}{|c|}{ Human samples } & \multicolumn{3}{|c|}{ Livestock } & \multirow[b]{2}{*}{ Wild animals } & \multirow[b]{2}{*}{ Ticks } & \multirow[b]{2}{*}{ Tota } \\
\hline & Acute hepatitis & Acute pneumonia & Chronic & Sheep & Goat & Cattle & & & \\
\hline $1+^{*}$ & & 1 & & 1 & & & & & 2 \\
\hline $11+$ & & & & 1 & & & & & 1 \\
\hline $\mathrm{III+}$ & & & & 5 & 3 & 7 & & & 15 \\
\hline $\mathrm{IV}+$ & & & & 1 & & & & & 1 \\
\hline IV- & 6 & & 12 & 5 & 1 & & 4 & & 28 \\
\hline $\mathrm{Vl}-$ & & & & & & & & 1 & 1 \\
\hline $\mathrm{VII+}$ & & & & & & & & 1 & 1 \\
\hline VII- & 3 & & & & & & & 31 & 34 \\
\hline VIII+ & & & 1 & 2 & 3 & & & & 6 \\
\hline VIII- & 1 & & & & & & & & 1 \\
\hline Total & 10 & 1 & 13 & 15 & 7 & 7 & 4 & 33 & 90 \\
\hline
\end{tabular}

*: plus and minus signs refer to adaA presence/absence, respectively.

In summary, from the theoretically possible $16 \mathrm{GT}$ ( 8 GG positive or negative for $a d a A$ ), 10 were identified in the samples studied (Table 2).

\section{Discussion}

A multiplex PCR coupled with hybridization by RLB for the characterization of $C$. burnetii was designed, allowing for its classification into the previously known 8 GG [15] and into up to 16 genotypes, depending on adaA presence/absence. For validation, 15 reference strains characterized in previous studies were used (Additional file 1: Table S1). All of them fell in the same GGs as previously described, when data was available, or grouped in the same clade as described [8$10,12,13]$. Consequently, an excellent correlation with some previously published schemes and, specifically, with the microarray-based whole genome typing of Beare et al. [15] was observed: the 4 isolates studied by Beare et al. that were also analyzed in this study (NMI, GG I; Henzerling, GG II; Priscilla, GG IV; and Scurry Q217, GG V) were classified with this method into the same GG as described. Also, the analysis of the results by InfoQuest disclosed a tree whose topology was similar to that of Beare et al. [15], with the

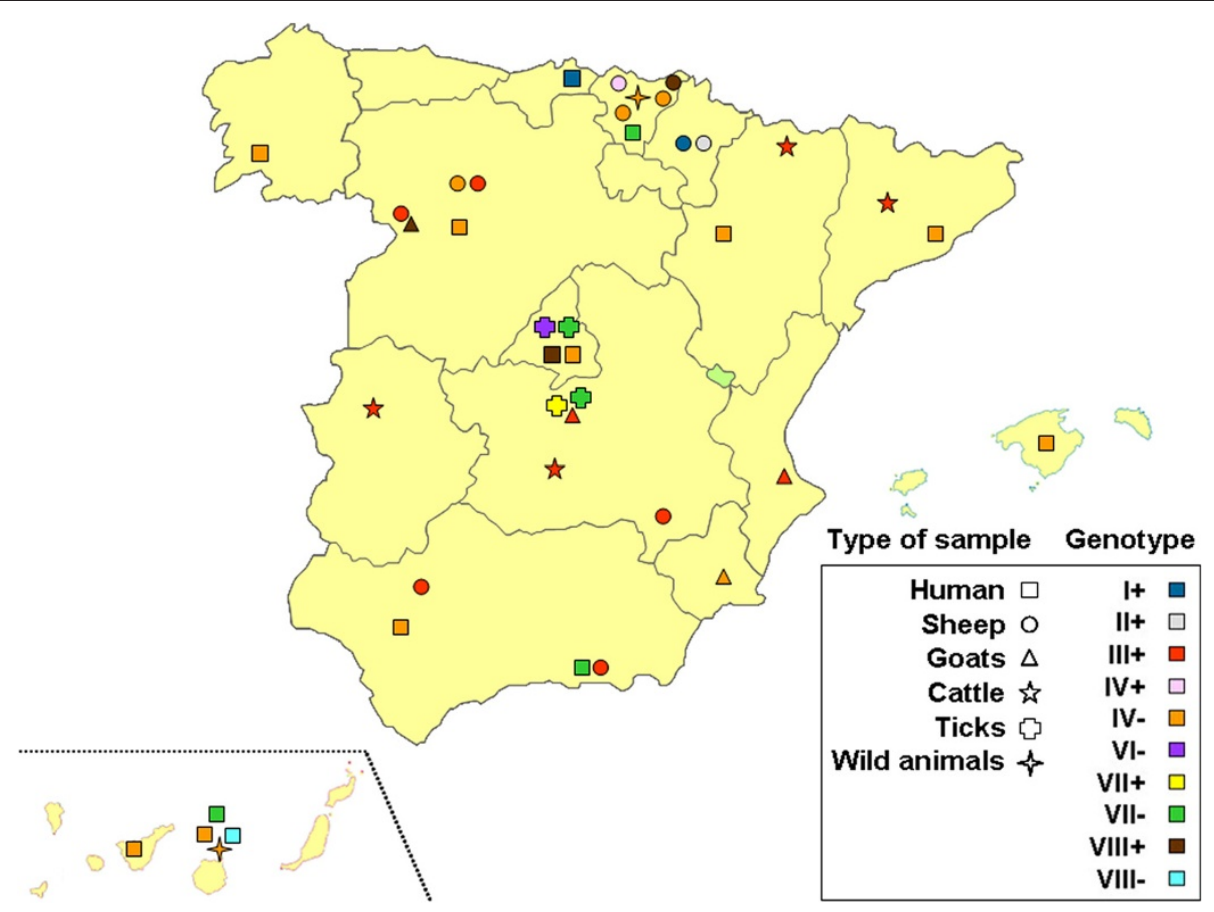

Figure 3 Map of Spain showing sampling sites, type of samples and results. 
only exception of GG VIII, which in this study grouped together with GGs I, II and III, instead of with GGs IV, V, VI and VII as in the Beare study.

With this methodology, a preliminary characterization of $C$. burnetii variants circulating in Spain has been performed showing a high variability of this organism in clinical and environmental settings, identifying $7 \mathrm{GG}$, with the exception of GG V, and 10 different GTs.

In Spain, while a respiratory disease is observed in about $80 \%$ of cases reported from the Northern region of the Basque Country [26,27], the Southern regions of Andalusia and the Canary Islands report a clear predominance (about 90\% of cases) of FID with liver involvement [28-34]. This last has also been described in Australia, France, Greece, or Taiwan [35-38], among others. Even taking into account the limited size of this study and the constraint of an extrapolation, a strain-associated factor that might explain the different clinical presentations of acute $Q$ fever is hypothesized for our country. The pattern observed in cases of acute $\mathrm{Q}$ fever indicates an association between absence of $a d a A$ and FID with liver involvement, produced in this study by adaA negative strains in both regions (the Southern regions of Andalusia and the Canary Islands), although is not statistically significant in this study $(p=0.09)$ due to low number of samples. Also, another sample of a case of hepatitis from the north (Basque Country) yielded an $a d a A$ negative result as well. The same applies for the 2 reference isolates from hepatitis cases analyzed in this study: F2, a French isolate and SQ217, recovered in the USA from a case of chronic hepatitis, are both adaA negative as well. In contrast, pneumonia predominates over liver involvement in Northern Spain, being the only case of this clinical form available for the study produced by an $a d a A$ positive strain. No other marker used in this study correlated with the clinical presentation of acute $Q$ fever. Availability of samples from cases of acute $Q$ fever for genotyping is much less frequent than from cases of chronic Q fever, even though acute Q fever is much more prevalent. In this study, 11 samples from acute cases were analyzed, although only one was from a case with respiratory symptoms, reflecting the limited availability of such samples, which may be due to a poor clinical awareness.

From the 10 GTs found in the country, only 5 have been detected in humans and, among them, GT IV- is the most frequently found in acute and chronic cases (75\% of cases). This GT has also been found in many mammal species (sheep, goat, wild boar and rats). Whether this could be interpreted as a higher tendency of this GT to cause illness in humans can not be inferred by this study, mainly considering that most of the acute cases (8/11) came from the same area (Gran Canaria Island). In any case, GT IV- is highly prevalent also in our chronic cases that came from 8 distant areas of the country, showing a more intensive circulation of this GT in humans.
The association previously proposed between adaAnegative strains and chronic disease [19] has been reproduced here for all chronic cases studied (13 cases), except for a sample from a vascular infection that was adaA positive. Beare et al. [15] hypothesized that the association between GG IV and chronic cases (as in 12 out of 13 chronic cases studied here) could be related to the slow growth of isolates from this genotype and, therefore, the induction of a decrease in the immune response. On the other hand, Zhang et al. hypothesized that adaA positive strains were related to acute cases [19], as it is the case of the only sample from a patient with acute pneumonia available. However, in our study, acute cases of FID with liver involvement were all produced by adaA negative strains.

GTs found in humans were also found in sheep, goats, rats, wild boar and ticks. This distribution of GTs suggests that sheep and goats are responsible for the transmission of C. burnetii to humans in Spain, as in other areas [39], and exhibit a high variability of GT. However, although in general domestic ruminants are important reservoirs for C. burnetii and play a relevant role in its transmission to humans, 4 of 24 human samples were found carrying GTs not found in ruminants in this work. A recent Spanish study [40] has also detected C. burnetii in roe deer, wild boar, carrion birds and hares. Although there is no data available on the genotyping of these specimens, more studies are needed to characterize the enzootic cycle of $C$. burnetii and its GT distribution in wildlife, as well as to ascertain whether other sources could be responsible for the transmission of C. burnetii to humans. GG VII was only found in ticks (H. lusitanicum, Dermacentor marginatus and Rhipicephalus sanguineus) and in 3 cases out of 10 of FID with liver involvement. It is to note that, while reference isolates from ticks belonged mostly to GG II, this GG has not been found in ticks in our study. Although the analyzed tick specimens came from 5 different areas, they were all from Central Spain, which could be biasing this data. Transmission of $\mathrm{Q}$ fever by tick bite still remains controversial $[41,42]$, and cases of simultaneous or consecutive infections with $C$. burnetii and other tick-borne agents have been described [43]. Whether C. burnetii can be transmitted by tick bite or not, the detection in ticks of GT VII-, found only in human patients revives this debate. More studies are needed to definitely clarify this question. On the other hand, given that GG VII isolates have not been found in cattle, sheep and goats in this study, we could think of other unknown reservoirs that could be involved as a source of infection of this GG for both ticks and humans. Traditional mammal species on which the tick species analyzed in this study feed on include rabbits (frequent all over Spain) for the immature stages of $H$. lusitanicum, which seems to be very important for the maintenance of populations of this tick species, small mammals 
for those of $D$. marginatus, and canids for all stages of $R$. sanguineus. Adult $H$. lusitanicum and D. marginatus normally feed on large ungulates. Animals present in the tick study areas included, apart from cattle, high densities of rabbits and other wildlife. It is to note that 40 liver samples from rabbits hunted in Gran Canaria analyzed by PCR were all negative (data not shown), although more studies are needed. Whether some of the above mentioned animals may act as reservoirs for GG VII C. burnetii remains to be studied.

Interestingly, in 7 cattle samples from 4 distant regions, only GG III was detected. In the study of Arricau-Bouvery [13] most of the cattle isolates (12/14) analyzed by MLVA also grouped together in a clade that is close but different to the one that include GG I isolates, as in this study. In Beare's study GG III is also philogenetically close to GG I and both clades appear together in the tree. This GG having never been found in humans in Spain so far lead us to hypothesize that cattle could represent a low risk for Q fever transmission to humans in our country.

One of the added values of the method described here is that it could be applied to any PCR-positive sample carrying at least 10 genome equivalents of the target organism, thus avoiding the need for culturing the organism to obtain data on the global circulation of $C$. burnetii. The frequent lack of human isolates from outbreaks, which are needed to apply the yet described methods, hamper a correct outbreak study that are necessary to identify the source of infection. This methodology allows the characterization directly from clinical samples avoiding the culture step of this fastidious bacterium, and proves to be valuable identifying so far 10 different GTs circulating in Spain. This method can be performed in any laboratory with basic equipment. It can easily determine relationships among $C$. burnetii from different origins by using PCR-positive samples, thus helping in the identification of the source of an outbreak in a rapid analysis.

\section{Conclusions}

The method described here is rapid, reproducible and sensitive. It can be applied directly to clinical and environmental samples, and is able to identify up to 16 GT. This will facilitate the acquisition of global data on the circulation of GT of this organism.

We have found a high variability of C. burnetii in Spain, with 10 GTs found in different settings, 5 of them in human samples. Interestingly, all the samples from acute cases of FID with liver involvement were produced by $a d a A$ negative microorganisms, while the only case of pneumonia available for the study was caused by a adaA positive strain. Moreover, the majority (12 cases) of the 13 chronic cases studied were produced by organisms of GG IV-, except for a case of vascular infection (GG VIII +).
Regarding livestock, human cases share GTs with sheep and goats, but the only GT found in cattle has never been found in humans.

The most frequent GT found in ticks (GT VII-; 31 out of 33 specimens studied) was also found in human samples of cases of FID with liver involvement. Although the hypothesis of transmission of $\mathrm{Q}$ fever by tick bite still remains controversial, to further study this point is of interest.

\section{Additional files}

Additional file 1: Table S1. Samples and reference isolates used in the study.

Additional file 2: Table S2. Oligonucleotides used in the study.

\section{Acknowledgements}

We thank Dr. Marco Quevedo, from the Institute of Virology, Bratislava, Slovakia, and Dra. Fatima Bacelar from the Centro de Estudos de Vectores y Doenças Infecciosas, Aguas de Moura, Portugal, for their help in setting up the culture method for C. burnetii, and Aleida Villa, from EXOPOL, Zaragoza, Spain, for providing local strains from livestock.

We are grateful to COST action B28 C05.0103 "Array technologies for BSL3 and BSL4 pathogens" for providing a platform of cooperation and for the exchanging of bacterial strains with other European laboratories, specifically with the Bundeswehr Institute of Microbiology, Munich, Germany (Dr. Dimitrios Frangoulidis) and the Institute of Virology, Slovak Academy of Sciences, Bratislava, Slovakia (Dr. Rudolf Toman).

Grant support for this work was from FIS PI10/00165, FUNCIS 26/03 from the Gobierno de Canarias "Diagnóstico directo de rickettsiosis prevalentes en nuestro medio (fiebre Q y tifus murino)", from the "Departamento de Agricultura y Pesca, Gobierno Vasco" "Ensayo de control de la fiebre Q en la cabaña ovina lechera de la CAPV", INIA FAU2006-00002-C04-01 to -04 "Ecología y control de la fiebre Q: Epidemiología molecular de Coxiella burnetii", and AGL2010-21273-C03-01-GAN from CICYT "Interacciones-inmuno endocrinas materno-fetal y con Coxiella burnetii en vacas lecheras de alta producción".

\section{Author details}

'Laboratorio de Espiroquetas y Patógenos Especiales, Department of Bacteriology, Centro Nacional de Microbiología, Instituto de Salud Carlos III, Ctra. de Pozuelo km 2.6, Majadahonda, Madrid 28220, Spain. ${ }^{2}$ Microbiology Service and Infectious and Tropical Diseases Unit, Hospital Universitario Insular de Gran Canaria, Av Marítima del Sur, s/n, 35016 Las Palmas, Spain. ${ }^{3}$ Department of Production and Animal Health, NEIKER - Instituto Vasco de Investigación y Desarrollo Agrario, Berreaga Kalea, 1, Derio, Bizkaia 48160, Spain. ${ }^{4}$ Department of Animal Health, Facultad de Veterinaria, Universidad Complutense de Madrid, Avda. Puerta de Hierro, s/n, Madrid 28040, Spain. ${ }^{5}$ Department of Animal Production, Universidad de Lleida, Pl. de Víctor Siurana, 1, Lleida 25003, Spain. ${ }^{6}$ Internal Medicine Service, Hospital Comarcal de Laredo, Avda. de los Derechos Humanos, s/n, Laredo, Cantabria 39770, Spain. ${ }^{7}$ Hospital Universitario Donostia and CIBERES, P० Doctor José Beguiristain s/n, Donostia-San Sebastián, Guipúzcoa 20014, Spain.

${ }^{8}$ Department of Animal Medicine and Surgery, Facultad de Veterinaria, Universidad de Las Palmas de Gran Canaria, Campus Universitario de Arucas s/n, Arucas, Las Palmas 35413, Spain. ${ }^{9}$ Present address: Department of Molecular Genetics and Microbiology, Center for Infectious Diseases, Stony Brook University, Stony Brook, NY 11794-5120, USA.

\section{Authors' contributions}

IJ, HG, RE and PA participated in the design of the study. CCR, MB, JLPA and NFR studied clinical and environmental samples from Canary Islands suspected of C. burnetii infection and provided the positives to the Centro Nacional de Microbiología-Instituto de Salud Carlos III (CNM-ISCIII) for molecular analysis. JFB, IA and ALGP studied livestock and tick samples from the Basque Country and provided the positives to the CNM-ISCIII for characterization. AT and ASO studied environmental samples from Madrid 
and provided the positives to the CNM-ISCIII for characterization. BS and FLG studied livestock samples from Catalonia and provided the positives to the CNM-ISCIII for molecular analysis. FPV and GC studied samples from Q fever patients and provided the positives to the CNM-ISCIII for molecular analysis. IJ, CGA and MRV participated in the culture and manipulation of the isolates in the BSL3 laboratory. IJ and PA designed the method of characterization. IJ, $R E, C G A, B L$ and MRV evaluated and carried out the genotyping method. HG and PA performed the phylogenetic analysis. IJ, HG, RE and PA participated in the interpretation of data and drafted the manuscript. All authors have critically read and approved the final version of the manuscript and also concur with further revisions of it.

Received: 31 October 2011 Accepted: 1 June 2012

Published: 1 June 2012

\section{References}

1. Raoult $\mathrm{D}$, Marrie $T$ J, Mege JL: Natural history and pathophysiology of $\mathrm{Q}$ fever. Lancet Infect Dis 2005, 5:219-226.

2. Rotz LD, Khan AS, Lillibridge SR, Ostroff SM, Hughes JM: Public health assessment of potential biological terrorism agent. Emerg Infect Dis 2002 8:225-230.

3. Minnick MF, Heinzen RA, Reschke DK, Frazier ME, Mallavia LP: A plasmidencoded surface protein found in chronic-disease isolates of Coxiella burnetti. Infect Immun 1991, 59:4735-4739.

4. Samuels JE, Frazier ME, Mallavia LP: Correlation of plasmid type and disease caused by Coxiella burnetii. Infect Immun 1985, 49:775-779.

5. Stein A, Raoult D: Lack of pathotype specific gene in human Coxiella burnetii isolates. Microb Pathog 1993, 15:177-185.

6. Nguyen SV, Hirai K: Differentiation of Coxiella burnetii isolates by sequence determination and PCR-restriction fragment length polymorphism analysis of isocitrate dehydrogenase gene. FEMS Microbiol Lett 1999, 180:249-254

7. Andoh M, Nagaoka H, Yamaguchi T, Fukushi H, Hirai K: Comparison of Japanese isolates of Coxiella burnetii by PCR-RFLP and sequence analysis. Microbiol Immunol 2004, 2004(48):971-975.

8. Hendrix LR, Samuel JE, Mallavia LP: Differentiation of Coxiella burnetii isolates by restriction-endonuclease-digested DNA separated by SDS-PAGE. J Gen Microbiol 1991, 137:269-276.

9. Jager C, Willems H, Thiele D, Baljer G: Molecular characterization of Coxiella burnetii isolates. Epidemiol Infect 1998, 120:157-164.

10. Glazunova O, Roux V, Freylikman O, Sekeyova Z, Fournous G, Tyczka J, Tokarevich N, Kovacava E, Marrie TJ, Raoult D: Coxiella burnetii genotyping. Emerg Infect Dis 2005, 11:1211-1217.

11. Mediannikov O, Fenollar F, Socolovschi C, Diatta G, Bassene H, Molez JF, Sokhna C, Trape JF, Raoult D: Coxiella burnetii in humans and ticks in rural Senegal. PLoS Negl Trop Dis 2010, 4:e654.

12. Svraka S, Toman R, Skultety L, Slaba K, Homan WL: Establishment of a genotyping scheme for Coxiella burnetii. FEMS Microbiol Lett 2006, 254:268-274.

13. Arricau-Bouvery N, Hauck Y, Bejaoui A, Frangoulidis D, Bodier CC, Souriau A, Meyer $\mathrm{H}$, Neubauer $\mathrm{H}$, Rodolakis A, Vergnaud G: Molecular characterization of Coxiella burnetii isolates by infrequent restriction site-PCR and MLVA typing. BMC Microbiol 2006, 6:38.

14. Roest HI, Ruuls RC, Tilburg JJ, Nabuurs-Franssen MH, Klaassen CH, Vellema P, van den Brom R, Dercksen D, Wouda W, Spierenburg MA, van der Spek AN, Buijs R, de Boer AG, Willemsen PT, van Zijderveld FG: Molecular epidemiology of Coxiella burnetii from ruminants in $\mathrm{Q}$ fever outbreak, the Netherlands. Emerg Infect Dis 2011, 17:668-675.

15. Beare PA, Samuel JE, Howe D, Virtaneva K, Porcella SF, Heinzen RA: Genetic diversity of the $\mathrm{Q}$ fever agent, Coxiella burnetii, assessed by microarraybased whole-genome comparisons. J Bacteriol 2006, 188:2309-2324.

16. Denison AM, Thompson HA, Massung RF: IS1111 insertion sequences of Coxiella burnetii: characterization and use for repetitive element PCR-based differentiation of Coxiella burnetii isolates. BMC Microbiol 2007, 7:91.

17. Huijsmans CJ, Schellekens JJ, Wever PC, Toman R, Savelkoul PH, Janse I, Hermans MH: Single-nucleotide-polymorphism genotyping of Coxiella burnetii during a $\mathrm{Q}$ fever outbreak in The Netherlands. Appl Environ Microbiol 2011, 77:2051-2057.

18. Hornstra HM, Priestley RA, Georgia SM, Kachur S, Birdsell DN, Hilsabeck R, Gates LT, Samuel JE, Heinzen RA, Kersh GJ, Keim P, Massung RF, Pearson T: Rapid typing of Coxiella burnetii. PLoS One 2011, 6:e26201.
19. Zhang G, To H, Russell KE, Hendrix LR, Yamaguchi T, Fukushi H, Hirai $K$, Samuel JE: Identification and characterization of an immunodominant 28-kilodalton Coxiella burnetii outer membrane protein specific to isolates associated with acute disease. Infect Immun 2005, 73:1561-1567.

20. Musso D, Raoult D: Coxiella burnetii blood cultures from acute and chronic Q-fever patients. J Clin Microbiol 1995, 33:3129-3132.

21. Toledo A, Olmeda AS, Escudero R, Jado I, Valcárcel F, Casado-Nistal MA, Rodríguez-Vargas M, Gil H, Anda P: Tick-borne zoonotic bacteria in ticks collected from central Spain. AmJTrop Med Hyg 2009, 81:67-74.

22. Willems $H$, Thiele $D$, Frolich-Ritter $R$, Krauss $H$ : Detection of Coxiella burnetii in cow's milk using the polymerase chain reaction (PCR). Zentralbl Vet $B$ 1994, 41:580-587.

23. Berri M, Laroucau K, Rodolakis A: The detection of Coxiella burnetii from ovine genital swabs, milk and fecal samples by the use of a single touchdown polymerase chain reaction. Vet Microbiol 2000, 72:285-293.

24. Barandika JF, Hurtado A, García-Esteban C, Gil H, Escudero R, Barral M, Jado I, Juste RA, Anda P, García-Pérez AL: Tick-borne zoonotic bacteria in wild and domestic small mammals in northern Spain. Appl Environ Microbiol 2007, 73:6166-6171.

25. Jado I, Escudero R, Gil H, Jiménez-Alonso Ml, Sousa R, García-Pérez AL, Rodríguez-Vargas M, Lobo B, Anda P: Molecular method for identification of Rickettsia species in clinical and environmental samples. J Clin Microbiol 2006, 44:4572-4576.

26. Montejo-Baranda M, Corral-Carranceja J, Aguirre-Errasti C: Q fever in the Basque Country: 1981-1984. Rev Infect Dis 1985, 7:700-701.

27. Montes M, Cilla G, Vicente D, Nieto V, Ercibengoa M, Perez-Trallero E: Gipuzkoa, Basque Country, Spain (1984-2004): a hyperendemic area of Q fever. Ann N Y Acad Sci 2006, 1078:129-132.

28. Alarcón A, Villanueva JL, Viciana P, López-Cortés L, Torronteras R, Bernabeu M, Cordero E, Pachón J: Q fever: epidemiology, clinical features and prognosis. A study from 1983 to 1999 in the South of Spain. J Infect 2003, 47:110-116.

29. Bolaños M, Santana OE, Pérez-Arellano JL, Ángel-Moreno A, Moreno G, Burgazzoli JL, Martín-Sánchez AM: Fiebre Q en Gran Canaria: 40 nuevos casos. Enferm Infecc Microbiol Clin 2003, 21:20-23.

30. Lepe JA, Guerrero FJ, Ruiz-Calderón A, del Castillo E, Gómez-Salvago S, Jiménez-Alonso MA, Palomo S, Perea R: Epidemiología de la fiebre Q en la zona norte de Huelva. Enferm Infecc Microbiol Clin 1999, 17:65-68.

31. Pascual-Velasco F, Borobio MV, González Z, Carrascosa M: Clinical presentation of acute $\mathrm{Q}$ fever in Lanzarote (Canary Islands): a 2-year prospective study. Scand I Infect Dis 1996, 28:533-534.

32. Rivero A, Zambrana JL, Pachón J: Fiebre de duración intermedia. Enferm Infecc Microbiol Clin 2003, 21:147-152.

33. Romero-Jiménez MJ, Suárez-Lozano I, Fajardo JM, Benavente A, Menchero A, de la Iglesia A: Hepatitis como manifestación única de la fiebre Q: características clínicas y epidemiológicas en 109 pacientes. Enferm Infecc Microbiol Clin 2003, 21:193-195.

34. Millán Mon A, Argany Fajardo A, Febles Bethencourt J, González Caloca C, Vento Remedios TE, Fernández Cabrera M: Fiebre Q en la isla de La Palma. Revisión de 35 pacientes. An Med Interna 1989, 6:527-530.

35. Derrick E: The course of infection with Coxiella burnetii. Med J Aust 1973, 1:1051-1057.

36. Tissot Dupont $H$, Raoult D, Brouqui P, Janbon F, Peyramond D, Weiller PJ, Chicheportiche C, Nezri M, Poirier R: Epidemiologic features and clinical presentation of acute $\mathrm{Q}$ fever in hospitalized patients: 323 French cases. Am J Med 1992, 93:427-434.

37. Gikas A, Kofteridis DP, Manios A, Pediaditis J, Tselentis Y: Newer macrolides as empiric treatment for acute $\mathrm{Q}$ fever infection. Antimicrob Agents Chemother 2001, 45:3644-3646.

38. Chang K, Yan JJ, Lee HC, Liu KH, Lee NY, Ko WC: Acute hepatitis with or without jaundice: a predominant presentation of acute $\mathrm{Q}$ fever in southern Taiwan. J Microbiol Immunol Infect 2004, 37:103-108.

39. Tissot-Dupont H, Amadei MA, Nezri M, Raoult D: Wind in November, Q fever in December. Emerg Infect Dis 2004, 10:1264-1269.

40. Astobiza I, Barral M, Ruiz-Fons F, Barandika JF, Gerrikagoitia X, Hurtado A, García-Pérez AL: Molecular investigation of the occurrence of Coxiella burnetii in wildlife and ticks in an endemic area. Vet Microbiol 2011, 147:190-194.

41. Cinco M, Luzzati R, Mascioli M, Floris R, Brouqui P: Serological evidence of Rickettsia infections in forestry rangers in north-eastern Italy. Clin Microbiol Infect 2006, 12:493-495. 
42. Pascual-Velasco F, Carrascosa-Porras M, Martínez-Bernal MA, Jado-García I: Fiebre Q tras picadura de garrapata. Enferm Infecc Microbiol Clin 2007, 25:360.

43. Rolain $J M$, Gouriet $F$, Brouqui $P$, Larrey $D$, Janbon $F$, Vene $S$, Jarnestrom $V$, Raoult D: Concomitant or consecutive infection with Coxiella burnetii and tickborne diseases. Clin Infect Dis 2005, 40:82-88.

doi:10.1186/1471-2180-12-91

Cite this article as: Jado et al: Molecular method for the characterization of Coxiella burnetii from clinical and environmental samples: variability of genotypes in Spain. BMC Microbiology 2012 12:91.

\section{Submit your next manuscript to BioMed Central} and take full advantage of:

- Convenient online submission

- Thorough peer review

- No space constraints or color figure charges

- Immediate publication on acceptance

- Inclusion in PubMed, CAS, Scopus and Google Scholar

- Research which is freely available for redistribution 